Review Article

\title{
Routine maternal depression screening in pediatrics
}

\begin{abstract}
Research on maternal depression has primarily been of focus during the postpartum period. However, research indicates that 1 in 10 mothers experience major depressive disorders throughout the lifespan of a child. Maternal depression can affect a child's growth and development beginning in the prenatal periods. This recognized public health concern has birthed many programs and initiatives to tackle this problem. Currently, routine maternal depression screening is limited and is not reimbursable to providers by most insurers if occurring outside of the postpartum period. An underused resource for maternal depression screening is the child's pediatrician. The pediatrician tends to be the provider the mother interacts the most in the child's first year of life. These providers have the potential to reinvent evidence based maternal screening guidelines that can improve maternal, child, and societal outcomes.
\end{abstract}

Keywords: maternal depression, children health disparities, routine screens by pediatricians
Volume 3 Issue 2 - 2015

Jennifer Petrea
Implementation Consultant, Insight Global, USA

Correspondence: Jennifer Petrea, Implementation Consultant, Insight Global, USA, Tel 704-953-4I59,

Email ivyfields84@gmail.com

Received: June 06, 2015 | Published: November 27, 2015
Abbreviations: CCSP, community care of Southern piedmont; HPM, health promotion model; CCNC, community care of North Carolina; MCO, managed care organization; LME, local management entity

\section{Problem statement}

The Professional Capstone Project is often one of the most intimidating portions of a nurse's scholarly advancement. However, such a project is advantageous for both the professional and focused population. This author's capstone project is grounded in community health nursing. "Nursing interventions at the community level are geared toward health promotion, disease prevention and creating environments that foster the highest level of functioning and quality of life for people". ${ }^{1}$

This author has experienced complex challenges surrounding the awareness of mental health disparities of single mothers outside of the postpartum period. Certain trends this author noticed while working as a Medicaid nurse case manager in a community pediatric clinic included a high incidence of maternal depression, low self-esteem, and non-adherence to children's healthcare and safety recommendations. "Maternal depression and anxiety are related to an increased risk of child behavioral problems and these children are at greater risk from their exposure to maternal depression and anxiety than their exposure to other maternal risk factors such as smoking, binge drinking and domestic violence". 2 Screening for maternal depression usually occurs most frequently in the prenatal and postnatal periods. However, research indicates that the prevalence of maternal depression is highest in the first three years of life. This indicates a need for pediatricians to utilize screening tools routinely at well visits to assess for maternal depression. The child's physician may be the first professional to learn of the infant- and child-rearing difficulties of a distressed mother.

The impact of unrecognized maternal depression has the chance of negatively impact on a child's growth, development, behavioral and psychosocial skills. It is likely that pediatricians do not screen because of a lack of knowledge of resources, time constraints, reimbursements or staffing. The choices pediatric providers make about whether to use a maternal screening are usually made on the premise of perceived value for the time invested. "The time spent helping depressed mothers function better may pay preventive dividends in the child's mental health and healthy development. ${ }^{3}$
Such factors have a variety of effects on the nursing profession. First, unidentified maternal depression can trigger both psychological and physiology influences in children. Public health nurses have the opportunity to assess patients and families in a variety of settings. Home visits, practice encounters and health clinics are ways nurses can assess, collect, plan, and implement measures for sustainable policies and procedures for maternal depression screening. Raising awareness and providing education to providers and practices can promote a reduction in maternal depression and the adverse effect it has on the pediatric population. The proposed solution would be to integrate maternal depression screening as a part of the well child visits. There would be no age limitations after age three, however, documentation of high-suspiciousness cases. Data collected through screening tools can facilitate the proof that more highly trained staff and resources are of need within the pediatric clinics.

\section{Incorporated theory}

The theory this author chose to incorporate to support the proposed solution to the chosen problem was Pender's health promotion theory. Also known as the Health Promotion Model (HPM), this model "was designed to be a "complementary counterpart to models of health protection". ${ }^{4}$ One construct that is deeply embedded in Pender's HPM is "perceived self-efficacy". This concept is described as "judgment of one's personal ability to carry out a particular course of action". 5

The rationale for using this theory with the proposed solution of routine maternal depression screening by pediatricians, is to appreciate that a child's health promotion and protection are dependent upon a multi-dimensional nature of persons they interact within their environment to pursue health. Maternal well-being and perceived efficacy directly affects the likelihood for maternal participation in primary health promotion activities. Health promotion is directed at increasing an individual's level of well- being. Children are dependent upon their primary caregivers to meet basic needs for survival. Maternal relationships are of focus in this author's capstone project because of the holistic influence they have on a child's outcomes. "Children's exposure to chronic maternal depression seems to be associated with more problematic outcomes for children, perhaps because depression interferes with mothers' ability to respond sensitively and consistently over time. ${ }^{6}$ If pediatricians or other primary care providers fail to assess the well-being of a child's primary caregiver, then it is evident that other efforts in primary health promotion will be counterproductive. 
Pender's Health Promotion Model will be used in this author's capstone to promote community driven initiatives to promote positive growth and development in children's health. Not only does the model expand the advocacy role of the nurse, by focusing on selfefficacy, it also puts the child's health in the hands of the mothers and pediatricians, allowing them to be agents of change. This model allows community stakeholders, providers, parents and nurses to work together towards a goal of a better quality of life for mothers, children and the community as a whole.

\section{Implementation plan}

A sound implementation plan can help align the vast amount of creative ideas with guidance and direction. Failure to integrate such plans can be costly and ensure dissatisfactory pursuit of acquiring change. This paper attempts to streamline an implementation plan for this author's professional capstone project.

Approval: It will be necessary to obtain approval from this author's leadership and executive management team. The supportive operational entity in this author's specialty is Community Care of Southern Piedmont (CCSP). CCSP is a non-profit managed care organization for one of the fourteen networks that fall under the governing body, Community Care of North Carolina (CCNC). CCNC is the managed care organization (MCO) that has coordinated and case managed select Medicaid programs of the state of North Carolina. Within each network, which is comprised of various counties, lies a large amount of community stakeholders. CCSP has a quality improvement team that operates under the guidance of CCNC's standardized plan. While CCNC sets foundational initiatives for evidenced based practice, CCSP is able to implement unique quality improvement programs through local grants. The most realistic starting point is to find a champion within the quality improvement program at CCSP. Therefore, buy-in must include CCSP's Medical Director, Executive Director, Quality Improvement Director, and Board of Directors, and must be strategically facilitated. This can be accomplished through careful planning, collaborating, and researching the problem area and leveraging this with realistic goals. This author is confident that approval would be granted if a well-written draft and Executive Summary is prepared for the time of presentation.

\section{Implementation plan}

A sound implementation plan can help align the vast amount of creative ideas with guidance and direction. Failure to integrate such plans can be costly and ensure dissatisfactory pursuit of acquiring change. This paper attempts to streamline an implementation plan for this author's professional capstone project.

Approval: It will be necessary to obtain approval from this author's leadership and executive management team. The supportive operational entity in this author's specialty is Community Care of Southern Piedmont (CCSP). CCSP is a non-profit managed care organization for one of the fourteen networks that fall under the governing body, Community Care of North Carolina (CCNC). CCNC is the managed care organization (MCO) that has coordinated and case managed select Medicaid programs of the state of North Carolina. Within each network, which is comprised of various counties, lies a large amount of community stakeholders. CCSP has a quality improvement team that operates under the guidance of CCNC's standardized plan. While CCNC sets foundational initiatives for evidenced based practice, CCSP is able to implement unique quality improvement programs through local grants. The most realistic starting point is to find a champion within the quality improvement program at CCSP.
Therefore, buy-in must include CCSP's Medical Director, Executive Director, Quality Improvement Director, and Board of Directors, and must be strategically facilitated. This can be accomplished through careful planning, collaborating, and researching the problem area and leveraging this with realistic goals. This author is confident that approval would be granted if a well-written draft and Executive Summary is prepared for the time of presentation. p. 189). After this period, the maternal mental health may not be addressed unless she has access to a primary care provider. The child's pediatrician is likely to be the healthcare provider that interacts most frequently with mothers in the child's first year of life. Nurses who work with mothers and children should be educated on maternal depression and the harmful effects it has on children's outcomes. Increasing awareness of this concern is primarily important to the nursing practice because of the negative consequences on health promotion.

\section{Evidence from literature review}

An exhaustive review of literature was completed on approximately 15 peer-reviewed journal articles. The research included a blend of literature and data analysis from various disciplinary viewpoints. Common trends amongst research articles included associations between maternal depression and children's health disparities, higher levels of parental stress in low-income families, lack of provider knowledge in navigating the mental health system for parents.

A key indicator throughout the literature was the fact that there is a correlation between maternal depression and increased complaints of the child's behavior. "Compared to children whose parents are not depressed, children of depressed parents have increased healthcare utilization, so the opportunities for intervention are readily available". The literature identified "red flags" that could aid in the identification of maternal depression; however, maternal depression is easily masked and is not mutually exclusive to visit characteristics. According to the research in the article by Heneghan, A., Silver, E., Bauman, L., \& Stein, R, "pediatricians may benefit from asking directly about maternal functioning or by using a structured screening tool to identify mothers who are at risk for developing depressive symptoms". These authors concluded that many pediatric providers did not recognized mothers who had self-reported high levels of depressive symptoms.

Nursing staff can help facilitate the dialogue between mothers and their child's provider by including screens into their functional health documentation. The literature suggests that many mothers did not report maternal depression or parental stressors because of fear of legal ramifications. However, the lack of community knowledge and parental trust in pediatric health promotion efforts can have counterproductive effects on children of society. "Mothers are, however, generally receptive to the idea of open communication with their pediatricians and are interested in receiving supportive written communication about parenting stress and depressive symptoms from pediatricians". 8

\section{Implementation logistics}

The most important early intervention to implement this change is to educate nursing staff who work in pediatric offices and interact with mothers at well child checks. CCSP's QI Specialist, who is also a Registered Nurse, will initially facilitate this. This intervention will be completed by an in-service on maternal depression. In order to assess nursing staff's knowledge and beliefs about maternal depression, a pre-test that involves a few questions about maternal depression will be administered to the nurses. After completion of the pre-test, 
an interactive Power Point presentation on maternal depression will be shown to the staff. This presentation will also review maternal depression screening tools and protocols. Upon completion of the presentation, a post-test will be given to assess knowledge gained from this opportunity. The next strategy to implement routine maternal depression screening by nurses at well child checks is to pilot this proposed practice on a small sample of mothers. The inclusion criteria for this sample will include the following:

1. The child must be an actively enrolled Medicaid recipient (Preferably with Carolina Access Case Management program for ease of access to data).

2. The child must be greater than or equal to 6 weeks of age.

3. The visit must be a scheduled, routine, well child check within the period between 6 weeks to 12 months of age.

\section{The child must be the mother's biological child}

In order to identify the children who fit into these criteria, a process with the scheduling and prior approval department must be identified. The QI Specialist will observe workflow in these departments and organize a tool to extract the data from the practice's scheduling database. Collaboration with information technology (IT) and EMR analyst will be helpful. The creation of an extraction tool will ensure that there is meaningful use of EHR and prevent further workload to be given to staff. Once the data is extracted from the scheduling database, the QI Specialist can communicate with the nursing staff and pediatricians to screen the mother of the scheduled child. One way to ensure this communication is to create a "flag" in the child's electronic health record. The mothers screening results will be given to the QI Specialist via completed paper screening tool. This will not be a part of the permanent health record. The QI Specialist will monitor the screens, provider interventions, referral process and completion. The QI Specialist will collaborate with the Local Management Entity (LME) that coordinates mental health services for NC Medicaid recipients to ensure completed referral and follow up if mother had a positive screen. The initial screen will be logged monthly and submitted to CCSP's financial department for provider reimbursement incentives. These incentives are usually funded by CCSP's QI funds that come from state and local grants. The incentives for provider participation will be as follows:

\section{1. $\$ 50.00$ for the initial maternal depression screen.}

2. $\$ 150.00$ for the completion of maternal follow-up after referral is made through the LME. For cost containment purposes, there will be a limit of 10 maternal depression screens completed weekly. This narrows the workload of the project team and staff. This program initiative will need approximately 3 years in order to appropriately collect and analyze data. The P-D-S-A cycle methodology will be used in order to assess program effects periodically.

\section{Resources needed}

1. Staff: QI Specialist from CCSP Managed Care Organization for audits and to serve as a liaison between clinic and Local Management Entity.

2. Educational Materials (pamphlets, handouts, posters, and PowerPoint presentations): Informational folder for staff: articles; pre \& post-test about maternal depression; possible competencies.

3. Assessment Tools (questionnaires, surveys, pre-and post- tests to assess knowledge of participants at baseline and after intervention); PHQ9

4. Technology (technology or software needs); possible collaboration with EHR analyst to incorporate screen in nursing documentation and to design data extraction tool to run queries of scheduling database

5. Funds (cost of educating staff, printing or producing educational materials, gathering and analyzing data before, during, and following implementation), and staff to initiate, oversee, and evaluate change.

6. The QI Specialist's salary will funded through a state grant, which is approximately $\$ 3,870.53$ to $\$ 4,704.27$ monthly wages. This has an annual cost of $\$ 56,451.24$; and a total program cost of $\$ 169,353.72$

7. The cost for the provider incentives will be directly related to their adherence with the conditions of participation. The initial maternal depression screen will return a $\$ 50.00$ incentive. $\$ 50.00$ $\mathrm{x} 10$ screens weekly $=\$ 500.00$ weekly expenditure; $\$ 500.00 \mathrm{x}$ 's 4 weeks $=\$ 2000.00$ monthly expenditure; $\$ 2,000.00$ monthly expenditure x's 52 weeks $=\$ 24,000.00$ annual program costs: Annual costs X's 3 years of program life $=\$ 72,000.00$ needed for provider reimbursements for initial maternal screen.

8. The additional incentive of $\$ 150.00$ for completed screen will carry a potential program cost of an additional $\$ 216,000$. The likelihood that every screen will result in referral to mental health services is rare. However, it is important to request these funds in proposed program budget in order to have some financial overhead if the Board of Directors reduces budget.

9. The cost for printed materials for the program life will be approximate to the potential to complete 1440 screens during program life. This number is 10 screens weekly for 3 years. A digital copy of the maternal screen (Edinburg, PHQ9) will be available to practice with reproduction rights. Approximately $\$$ 200.00 will be budgeted for this.

10. The cost of temporary HIT consultant contract will be approximately $\$ 12,000.00$ over the program life.

11. Total estimated cost of program: \$ 493,553.00, however, adjustment for realistic sampling would suffice for a budget of $\$ 425,000.00$.

\section{Communications Strategies}

In order to implement this plan, a project team will be assigned to various duties and a timeline will be implemented. The project team will include: Project Manager, QI Specialist, Provider Liaison, and CCSP Nursing Case Management. Other stakeholders can join upon approval. There will be weekly meetings minimum during phase 1 . Follow up meetings will be planned as project runs its' course. The phases of this project are as follows:

1. Phase 1: This phase will involve the first 6 months after start date. This phase is for procedural adjustments and initial phase of data collection. The PDSA tool will be used to assess if initiatives and interventions are relevant and effective for change.

2. Phase 2: This phase will begin after the Phase 1 graduation date. This phase will include the process of streamlining data extraction tool, EMR integration and mental health referral protocols. This phase will run for approximately 20 months. 
3. Phase 3: This phase will include 6 months of data analysis.

4. Phase 4: This phase will be the last 4 months of the program. This phase will be used to evaluate performance measures and effectiveness.

\section{Evaluation process}

Outcomes of nursing care must be shown to relate to the specific care aspects of the process change (Frisch \& Kelley, 2002). "In general, there are three reasons why evaluations are conducted: to determine plausibility, probability, or adequacy" ("Purposes of Evaluations (Plausibility, Probability, Adequacy) - Unite For Sight", 2013, para, 1). A 6-month pilot, known as Phase 1 will be completed to test the efficacy and feasibility of a process change related to nursing assessment of maternal depression at well child checks in the child's pediatric clinic. The PDSA cycle format will be utilized to establish baseline data and allow for continuous improvement throughout project life. Several methods will be used to gather baseline data. Using a mix of data collection tools will allow for a broader sample to analysis throughout the project.

\section{Methods}

1. Survey of maternal attitudes and beliefs about discussing depressive symptoms with their child's pediatrician (prior to implementation of proposed solution).

2. Survey nursing staffs' beliefs about the importance of maternal depression screening (pre-education period).

3. Compare visits characteristics of those with identifiable maternal depression risk factors (throughout project).

4. Present case studies and presentation on maternal depression and effects on child's development.

5. Conduct survey of nursing staff's experience once process and workflow is past phase

\section{Variables to Consider Throughout The Project will Include, but not Limited to}

a. Nursing staff attitudes and perceptions about liabilities and scope of practice related to mental health disparities.

b. Patient attitudes and perceptions related to social stigmas and psychosocial factors related to maternal depression.

c. Assurance of mother accessing a primary care and mental health provider if screen is positive.

d. Challenges related to the navigation of the mental health system.

e. Barriers that prevent routine depression screening.

f. Willingness of mothers to participate.

Baseline data prior to project initiation will be collected through a random survey of mothers of the children seen at the pediatric clinic. These surveys will be presented to mothers at check in and will be turned in to front office prior to being seen by provider. This will be implemented prior to implementation of proposal.

Data collection for this evaluation process will be completed through chart extractions completed by the QI Specialist. In order to identify the mothers who fit inclusion criteria, a process with the scheduling and prior approval department must be identified. Commonly, the billing department verifies insurance and eligibility prior to a well-child check. This could be a helpful resource when screening Medicaid enrolled children. The QI Specialist will observe workflow in these departments and organize a tool to extract the data from the practice's scheduling database. Collaboration with information technology (IT) and EMR analyst will be helpful. The creation of an extraction tool will ensure that there is meaningful use of EHR and prevent further workload to be given to staff. Once the data is extracted from the scheduling database, the QI Specialist can communicate with the nursing staff and pediatricians to screen the mother of the scheduled child. One way to ensure this communication is to create a "flag" in the child's electronic health record. The mothers screening results will be given to the QI Specialist via completed paper screening tool. This will not be a part of the permanent health record. The QI Specialist will monitor the screens, provider interventions, referral process and completion. The QI Specialist will collaborate with the Local Management Entity (LME) that coordinates mental health services for NC Medicaid recipients to ensure completed referral and follow up if mother had a positive screen. The initial screen will be logged monthly and submitted to CCSP's financial department for provider reimbursement incentives.

For cost containment purposes, there will be a limit of 10 maternal depression screens completed weekly.

\section{Educational tools}

The educational tools and surveys used in this project are attached to assignment portal (Figures 1-6).

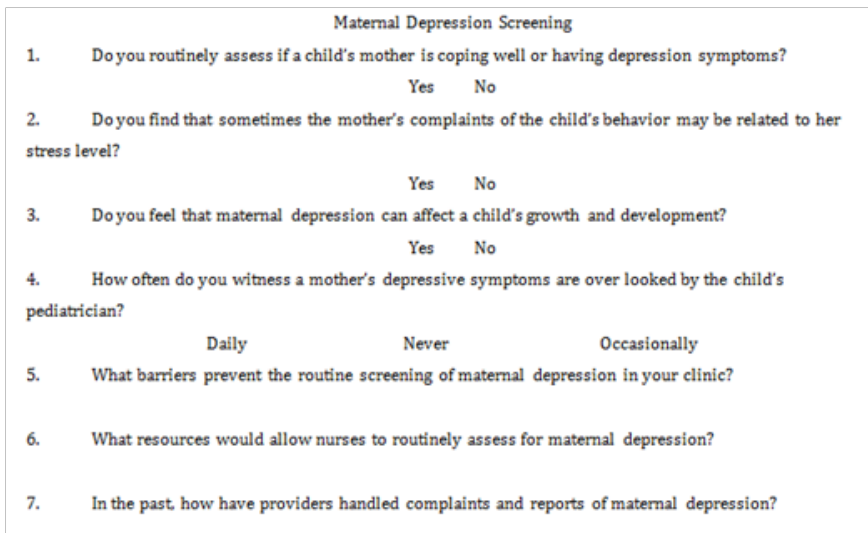

Figure I Survey of nursing.

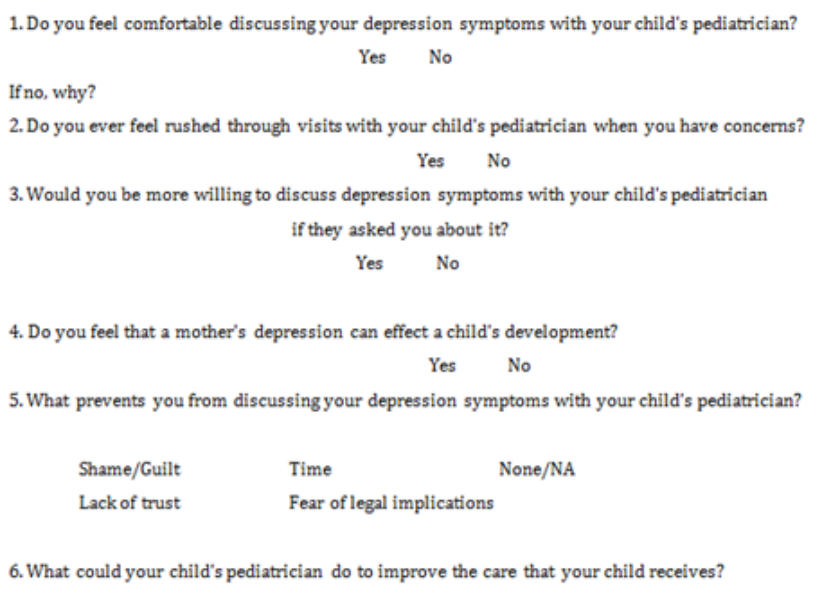

Figure 2 Survey for mothers. 
Suzanne Theberge, Project THRIVE at

AMCHP March 2007
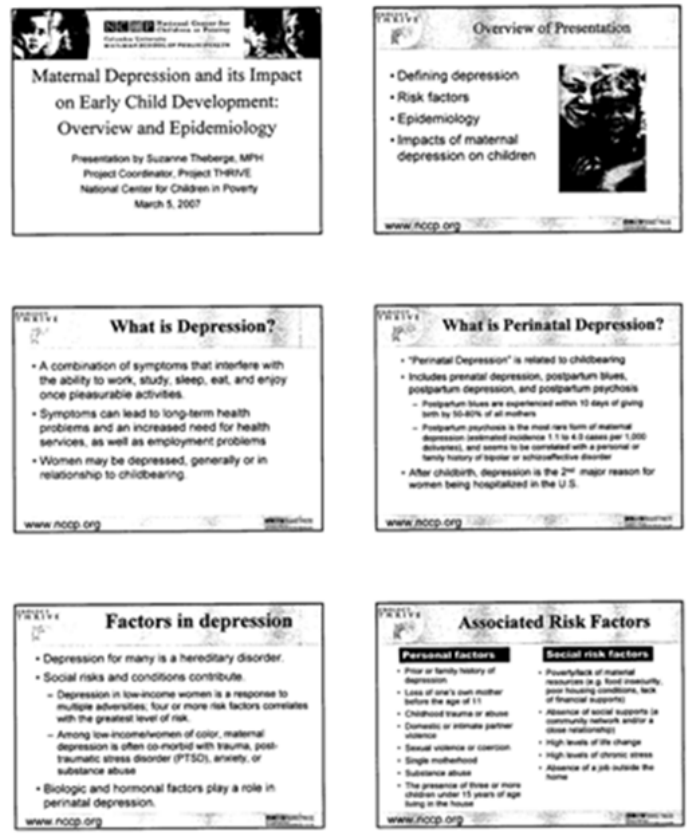

Reducing Matemal Depression and its Impact on Early Childhood Development

Figure 3 Reducing maternal depression.
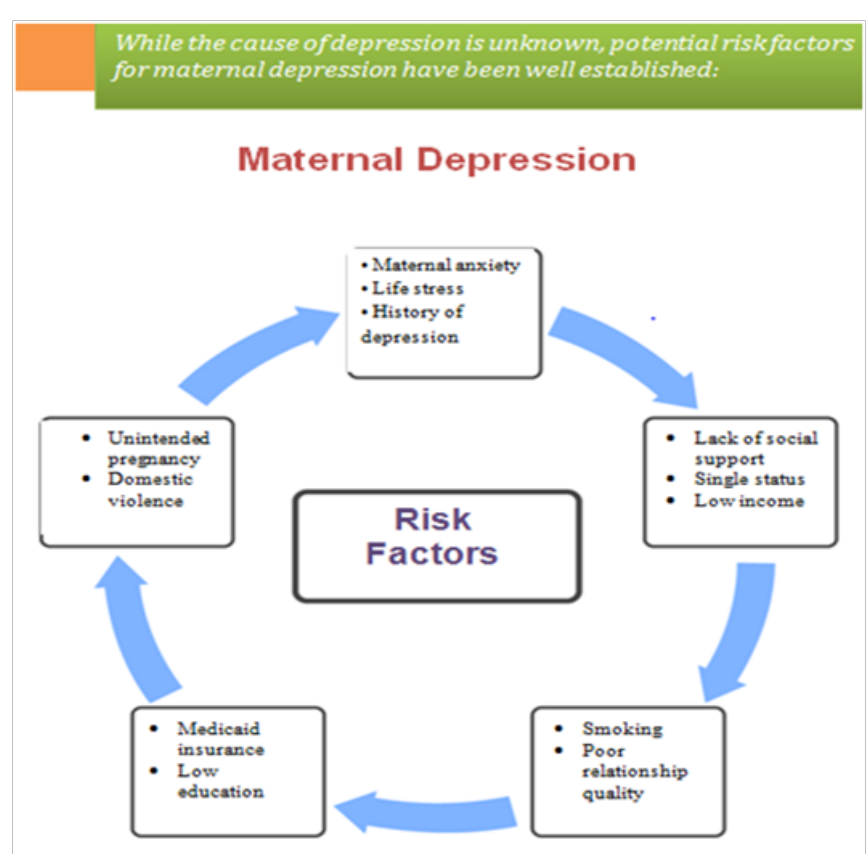

Figure 4 Maternal depression.

Performance based measures will be key into evaluating practice change. In order to do this, e the PDSA Cycle will be used throughout program to test for continuous and quality improvement. A worksheet will be utilized for baseline data testing. A new PDSA cycle will be the framework for each phase implementation. PDSA tests for small changes throughout a project life and can help facilitate cost containment.

Howand Why?

- As a pediatric nursing professional, you may be the first to recognize signs of depression in a mothe

- Screening tools can help introduce the subject of depression to a child's mother

- Screens can incorporated into the routine assessments at Well Child Checks

- Screening is an easy, quick, and affordable method of identifying women who may be struggling with depression

- Screening does not diagnose, but helps to identify at risk mothers who are and in need of further intervention or referral to mental health services

- Maternal depression has severe effects on a growing child's development

Barriers to Screening

- Mothers may not always recognize that common physical symptoms such as fatigue, lack of energy. poor sleep, and loss of appetite can mask depression.

- A mother who recognizes that she has symptoms of depression may be reluctant to discuss her symptoms with her child's provider because of shame, fear of legal action, and/or lack of energy

- Mothers should be encouraged by nursing staff to be open about their feelings, to seek help, and to feel that depression is not shameful and does not make her a bad mother.

- Many mothers may delay acknowledging the symptoms of depression or seeking help in hopes that the symptoms will pass with time, not realizing that time may just exacerbate their condition.

- Women should be informed about the effects of toxic stress on children's growth and development

- Many pediatric providers view navigating the mental health system as challenging

- There is often a lack of awareness and knowledge of mental health services

Reality

- Depression can occur across all age, race, ethnic and economic groups, every mother should be screened and educated about maternal depression.

If you recognize signs of depression in one of your patients, ask her about them and reassure her that help exists and she is not alone. Your screening and intervention could make all the difference in the world to women experiencing maternal depression, and to their families.

Figure 5 Information on screening for maternal depression.

\section{PATIENT HEALTH QUESTIONNAIRE (PHQ-9)}

\begin{tabular}{|c|c|c|c|c|}
\hline \multicolumn{2}{|l|}{ NAME:- } & \multicolumn{3}{|l|}{ DATE:- } \\
\hline \multirow{3}{*}{$\begin{array}{l}\text { Overt the last } 2 \text { woeks, now ofien have you been } \\
\text { botheres by any of the following problems? } \\
\text { (use } v \text { "to indicate your answer) }\end{array}$} & & & & \\
\hline & Not at all & $\begin{array}{c}\text { Several } \\
\text { days }\end{array}$ & $\begin{array}{c}\text { More than } \\
\text { natt the } \\
\text { days }\end{array}$ & \begin{tabular}{|l|l|l} 
Nearty \\
every day
\end{tabular} \\
\hline & 0 & 1 & 2 & 3 \\
\hline 2. Feeling domn, depressed, or hopeless & 0 & 1 & 2 & 3 \\
\hline 3. Trouble falling or staying asleep, of sleeping too much & o & , & 2 & 3 \\
\hline 4. Feeting trect or having ivele energy & 。 & 1 & 2 & 3 \\
\hline s. Poor appetite of overeation & 。 & 1 & 2 & 3 \\
\hline $\begin{array}{l}\text { 6. Feeting bas about yourselt- or that you are a tallure of } \\
\text { have tet yoursett of your family cown }\end{array}$ & 。 & 1 & 2 & 3 \\
\hline $\begin{array}{l}\text { 7. Troudte concentrating on things, such as reasing ine } \\
\text { nemspoper of watching television }\end{array}$ & 。 & 1 & 2 & 3 \\
\hline $\begin{array}{l}\text { 8. Moving or speaking so slowly that other people could } \\
\text { have noticed. Or the opposite - being so figety or } \\
\text { restless that you have been moving acound a lot more } \\
\text { than usuat }\end{array}$ & 。 & 1 & 2 & 3 \\
\hline \multirow[t]{2}{*}{ 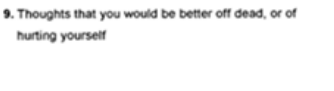 } & $\circ$ & 1 & 2 & 3 \\
\hline & sos columms & & . & \\
\hline \multicolumn{5}{|c|}{ 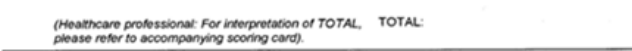 } \\
\hline \multirow{4}{*}{\multicolumn{2}{|c|}{$\begin{array}{l}\text { 10. If you checked oft any probsems, how dimour } \\
\text { have these problems made in for you to do } \\
\text { your work take care of things at home, or get } \\
\text { along with other people? }\end{array}$}} & \multirow{4}{*}{\multicolumn{2}{|c|}{ 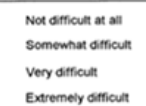 }} & $\overline{-}$ \\
\hline & & & & \\
\hline & & & & \\
\hline & & & & \\
\hline
\end{tabular}

$1263381004+2005$

Figure 6 Patient health questionnaire. 


\section{Disseminating evidence}

Perhaps one of the most critical parts of research is the dissemination of evidence. A researcher can have all the right implementation strategies, literature reviews, and evaluation plan, but if the dissemination of evidence is weak, then the project returns null and void. "Diffusion is the process by which an innovationwhether an idea, practice, or object that is perceived to be new by the adopter-is communicated through certain channels over a given period among the members of a social system". ${ }^{9-14}$ With that in mind, the following dissemination strategies are from guidance of The Commonwealth Fund's 2010 guidelines. Though The Commonwealth Funds guidelines are based on national quality campaigns, this author appreciates the structural and conceptual framework it provides.

Strategy 1: Highlight evidence base and relative simplicity of recommended practices

This author feels that one of the main barriers to routine maternal depression screens in pediatric offices is due mainly in part to the complexity of navigating the mental health system. Often, making routine referrals for their own patients can prove to be a daunting task. The take-away of this project is that the nursing profession can be advocates for mothers by assessing for maternal depressive symptoms during routine visits at the pediatrician's office.

Strategy 2: Align the campaign with the strategic goals of the adopting organizations

In order to get support for this project, it will be necessary to research what other organizations are strategically doing in the scope of proposed practice change. Careful assessment of community stakeholders and learning their programs and initiatives can help foster the proposed change.

Strategy 3: Increase recruitment by integrating opinion leaders into the enrollment process and employing a nodal organizational structure

To be influential in facilitating practice change, it is of great value to network and seek out the leaders in local public health administration. Inquiring and seeking their opinions can help build stronger network of support.

\section{Strategy 4: Form a coalition of credible campaign sponsors}

In this particular project, this author will network with colleagues from local health department, public health authority, LME, Social Services, Managed Care Organizations, Educational Health, and the local hospital system. The leaders of greatest importance will include pediatricians, nurses, obstetricians, and caseworkers.

\section{Strategy 5: Generate a threshold of participating organizations that maximizes network exchanges.}

This can be facilitated by networking and staying abreast to the results of community health assessments and initiatives. Knowing what others are doing can help pinpoint champions.

\section{Strategy 6: Develop practical implementation tools and guides for key stakeholder groups}

This strategy can be carried out successfully by knowing the roles and functions within certain stakeholder organizations. For example, knowing the contact that can consult on reimbursement procedures at the state level would be helpful.

\section{Strategy 7: Create networks to foster learning opportunities}

In addition to researching the proposed problem, it is important to frame the proposed solution with local context in order to innovate change within a network of stakeholders. Frequent community stakeholder meetings, poster announcements and mass communication can help to disseminate research findings.

\section{Strategy 8: Incorporate monitoring and evaluation of milestones and goals}

It is important to share with supportive stakeholders the information gained throughout the course of a project. This will allow for enhanced recommendations of areas involved with proposed solution and strategy. In this case, updating the LME of the baseline data may facilitate their involvement with processes and workflows to allow practice change.

\section{Review of literature}

According to Bassani et al. ${ }^{15}$ Estimating the number of children exposed to parental psychiatric disorders through a national health survey. Adolescent Psychiatry Mental Health.

a. Summary of Article: This article concluded that children with parents that have psychiatric problems are a higher risk of experiencing and being diagnosed with psychiatric disorders. These children also have prevalent rates of mortality and developmental problems.

b. Research Elements: Design, Methods, Population, Strengths, Limitations: The number of children that are exposed to parental psychiatric conditions was calculated using a design-based analysis. Data was obtained from national survey data to estimate how many children potentially are exposed to environments involving parental psychiatric disorders. Inclusion criteria for data collection included children that were at 12 years old living in the home, and the type of relationship the respondent had with the children. Variables such as parent-child relations and single parenthood were identified in order to calculate data.

c. Outcome(s): Research Results: In households in which those who responded to the survey and met criteria for one or more mental health disorder in the previous 12 months, nearly 570,000 children under 12 years old, or $12.1 \%$, and were exposed to parental psychiatric disorder. Almost $75 \%$ of these children have parents that report receiving no mental health care prior to the survey. Limitations include that the number of children exposed may be underestimated.

d. Significance to Nursing and Patient Care: The grossly stark amount of children exposed is a major public health concern which indirectly correlates with health disparities of children. Nurses must be aware that children exposed to toxic stress are at risk of having accidents, morbidity, mortality, and are at increased risk of developing psychiatric disorders. At well check appointments, nursing assessment can include screening for risk factors. Nurses can promote further research and engage in discussions for public health policy and strategic planning committees.

Barlow J et al. ${ }^{16}$ Parent-training programs for improving maternal psychosocial health. Cochrane Database System Review.

a. Summary of Article: This review expands on the evidence of reported short-term benefits of parenting programs on mental health outcomes and satisfaction with the partner relationship.

b. Research Elements: Design, Methods, Population, Strengths, Limitations: Rigorous methods were used by the impartial and independent Cochrane Collaboration to summarize collected data from literature reviews. Electronic databases were searched 
for randomized controlled trials in which participants had been allocated to an experimental or a control group, and there was at minimum, one standardized measure of parental mental and psychosocial health. Researchers used approximately 48 studies in this research. There were 4,937 participants involved and covered three types of programs: behavioral, cognitive-behavioral and multimodal. Limitations included limited studies reported father's outcomes separately. Though this data was limited, it showed statistically significant short-term improvements in paternal stress but did not conclude if these programs were beneficial in relation to the improvement of depressive symptoms, self-confidence or satisfaction with partner.

c. Outcome(s): Research Results: Overall, the researchers concluded that data analysis of focus group parenting programs led to statistically significant short-term improvements in mental health variables and factors. Stress and confidence were the only mental health variables reported with significance to statistics at six month follow-up, and none were significant a year later. There was no evidence of any effects on self-esteem at follow up. There were not any trials that reported aggression or adverse effects.

d. Significance to Nursing and Patient Care: The results within this research support that interventions such as parenting programs can help improve mental health in parents. In order to allocate such resources, an assessment of parental mental health must be done. Nurses can help identify risk factors and report parental statements to child's primary care physician.

Fortney, et al. ${ }^{17}$ says that Population level effectiveness of implementing collaborative care management for depression. General Hospital Psychiatry.

a. Summary of Article: Care management is feasible to provide in routine care, and the depression outcomes of patients reached by this evidence-based practice are similar to those observed in randomized controlled trials. According to this article, no studies have estimated effectiveness of care management at the population level when deployed in routine care.

b. Research Elements: Design, Methods, Population, Strengths, Limitations: This was a "multisite hybrid Type 3 effectivenessimplementation study" that employed a pre-post, quasiexperimental design. The population for sample included 22 Veterans Affairs community-based outpatient clinics. One variable assessed was medication possession ratios. The adherence index was determined for 1558 patients with current prescription for an antidepressant. Differences in treatment response rates at implementation and control sites were estimated from observed differences in MPR.

c. Outcome(s): Research Results: "Reach" into the target population at care management implementation sites was $10.3 \%$. Patients at care management implementation sites had a significantly higher probability medication adherence than patients at control sites (no care management).

d. Significance to Nursing and Patient Care: This data is significant to nursing because implementing nurse case managers in pediatric offices can help improve adherence to prescribed treatments and improve a child-centered plan of care.

According to Hanks $\mathrm{C}^{18}$ Health disparities research and service learning.

1. Summary of Article: The objective of this study was to involve a group of undergraduate students in social science research. The researchers sought to assess the response to community needs.
2. Research Elements: Design, Methods, Population, Strengths, Limitations: This study used narrative data collected from a focus group that included students and a group of Research.

3. Elements: Design, Methods, Population, Strengths, Limitations: This was a "multisite hybrid Type 3 effectiveness-implementation study" that employed a pre-post, quasi-experimental design. The population for sample included 22 Veterans Affairs communitybased outpatient clinics. One variable assessed was medication possession ratios. The adherence index was determined for 1558 patients with current prescription for an antidepressant. Differences in treatment response rates at implementation and control sites were estimated from observed differences in MPR.

4. Outcome(s): Research Results: "Reach" into the target population at care management implementation sites was $10.3 \%$. Patients at care management implementation sites had a significantly higher probability medication adherence than patients at control sites (no care management)

5. Significance to Nursing and Patient Care: This data is significant to nursing because implementing nurse case managers in pediatric offices can help improve adherence to prescribed treatments and improve a child-centered plan of care.

According to Hanks $\mathrm{C}^{18}$ Health disparities research and service learnin.

a. Summary of Article: The objective of this study was to involve a group of undergraduate students in social science research. The researchers sought to assess the response to community needs.

b. Research Elements: Design, Methods, Population, Strengths, Limitations: This study used narrative data collected from a focus group that included students and a group of Research Elements: Design, Methods, Population, Strengths, Limitations: Sampling included mothers from 5 community-based pediatric practices and 1 hospital-based practice to ensure a diversity of population sampling. A trained facilitator, who is experienced in conducting focus groups used open-ended questions and administered a questionnaire. Audiotapes, surveys, and transcripts of the focus groups were reviewed for major themes by 3 independent researchers. This research included grounded theory and immersion/crystallization techniques.

c. Outcome(s): Research Results: Using demographic variables of mothers from seven focus groups, researchers identified three themes. These themes were emotional health, self-efficacy, and support systems. Data suggested that most mothers were aware that their mental health was influential on their child's development. In all socioeconomic groups, mothers felt that if they talk about such issues with child's doctor, they would fear judgment and possible referral to child protective services. A stark number of mothers were willing to openly discuss these issues with child's pediatrician if they were more personally acquainted with them.

d. Significance to Nursing and Patient Care: The data in this article is important to nursing for several reasons, but namely the need for improved parent education. Nurses can initiate such conversations by asking about maternal stress and correlate reports of child's health accordingly.

Heneghan A et al. ${ }^{19}$ says that Do pediatricians recognize mothers with depressive symptoms.

a. Research Elements: Design, Methods, Population, Strengths, Limitations: Sampling included mothers from 5 community- 
based pediatric practices and 1 hospital-based practice to ensure a diversity of population sampling. A trained facilitator, who is experienced in conducting focus groups used open-ended questions and administered a questionnaire. Audiotapes, surveys, and transcripts of the focus groups were reviewed for major themes by 3 independent researchers. This research included grounded theory and immersion/crystallization techniques.

b. Outcome(s): Research Results: Using demographic variables of mothers from seven focus groups, researchers identified three themes. These themes were emotional health, self-efficacy, and support systems. Data suggested that most mothers were aware that their mental health was influential on their child's development. In all socioeconomic groups, mothers felt that if they talk about such issues with child's doctor, they would fear judgment and possible referral to child protective services. A stark number of mothers were willing to openly discuss these issues with child's pediatrician if they were more personally acquainted with them.

c. Significance to Nursing and Patient Care: The data in this article is important to nursing for several reasons, but namely the need for improved parent education. Nurses can initiate such conversations by asking about maternal stress and correlate reports of child's health accordingly.

Heneghan A et al. ${ }^{19}$ says that Do pediatricians recognize mothers with depressive symptoms.

1. Summary of Article: The researchers of the study in this article sought to determine if primary pediatric health care providers recognize depressive symptoms in mothers. Another aim of this study was to explore to the extent in which provider, maternal and visit characteristics affect the providers' ability to recognize mothers with depressive symptoms.

2. Research Elements: Design, Methods, Population, Strengths, Limitations: This study was completed using a cross-sectional analysis. It was conducted at a hospital located in an inner-city, pediatric clinic. There were two focus groups of mothers chosen to complete questionnaires. Participants were unaware of the other's responses and the questionnaires consisted of social and demographical variables. A self-administered assessment tool was used for mothers and screen depressive symptoms. The reported data from maternal sources was crossed referenced with provider assessments and commonalities were derived.

3. Outcome(s): Research Results: 214 participants, ages 15-45 years, were assessed by 60 pediatric providers. Common themes emerged from data showed that mothers primarily were single, black, hispanic, had a mean age of 26 years, and roughly $25 \%$ of these mothers lived alone. Of this study, approximately $40 \%$ of mothers had screens that represented high symptom depression levels. Out of 86 mothers, 25 of them were identified by pediatric providers. Using socio-demographic variables such as mothers who were $<30$ years old, receiving public assistance and living alone pediatric providers were more likely identify likely positive screens.

4. Significance to Nursing and Patient Care: The significance of the data is that it suggests that a child's provider often do not recognize mothers with positive self-reported maternal depression screens. Nurses can benefit from asking about maternal functioning directly or by using evidenced based screening tools to help identify those who are at risk for maternal depression. In addition, a nursing assessment inclusive of maternal depression screening can be an important augmentation to a referral process flow for those mothers who have children seen in pediatric offices.

Houtrow A et al. ${ }^{20}$ Pediatric mental health problems and associated burdens on families.

Summary of Article: This study reported that nearly 20\% of children in the United States have mental health disorders. It is not well understood how the factors associated with childhood mental health concerns and the burdens impact associated families. The goals of these researcher included profiling children with mental health problems, to identify related disparities, and to quantify the identification of family burdens.

1. Research Elements: Design, Methods, Population, Strengths, Limitations: For this study, researcher used data from the 2003 National Survey of Children's Health. For this analysis, the sample consisted of 85,116 children whose ages were 3-17 years.

2. Outcome(s): Research Results: Of the children aged 3-17 years, $18 \%$ had reported mental health problems. Of this group of children, $28 \%$ reported family burdens.

3. Significance to Nursing and Patient Care: Childhood mental health disorders are common and disproportionally affect children and family with fewer health care resources. Nurses can provide up to date community resources to families with children who have mental health problems. Nurse who advocate for families at highest risk for mental health problems and family burden will help assist in clinical improvement and policy makers to help improve support systems for children and families.

Jordal $\mathrm{M}$ et al. ${ }^{21}$ Unmarried women's ways of facing single motherhood in Sri Lanka - a qualitative interview study.

a. Summary of Article: This unique study looked at unmarried women in Sri Lanka who were facing single motherhood. The data allowed reflection on how these women utilize resources of their limited social environments, which are characterized by a lack social and financial support. These variables are often shown to have negative consequences for the health and well-being of both mothers and their children. The objective of this study was observe how these women handled their complex psychosocial situations.

b. Research Elements: Design, Methods, Population, Strengths, Limitations: This was a qualitative study of 28 unmarried, pregnant, women or single mothers and comprised of interviews that were semi-structured.. The researchers used qualitative content analysis to carry out this qualitative study and relate the results with the conceptual framework of social navigation.

c. Outcome(s): Research Results: The women in this study were facing single motherhood expressed awareness of making decisions that are often outside of societal norms. These women often reported attempt or consideration of suicide because of their feelings of shame and guilt they harbored. It appeared that these women suffered from self and societal injustices related to sexual immorality. The interviews revealed that the women expressed fear of shame, rejection towards familial and societal acceptance and they worried about financial survival.

d. Significance to Nursing and Patient Care: The data in this study can aid the nursing practice as social norms and cultural diversity is embraced. Nurses who are educated on the practices of ethnic and cultural groups can assess their patient's needs in multiple 
areas of service. Understanding the roles of mothers in cultural groups can help promote women's rights and health during childbearing year

Melnyk, et al. ${ }^{22}$ Creating opportunities for parent empowerment: program effects on the mental health/coping outcomes of critically ill young children and their mothers.

a. Summary of Article: The purpose of this study sought to evaluate the effects of preventive educational-behavioral intervention programs initiated early in the care of critically ill children requiring acute inpatient care. The study reviewed the effects of the program on the mental health/psychosocial outcomes these children and their mothers.

b. Research Elements: Design, Methods, Population, Strengths, Limitations: This study used a randomized, controlled trial with follow-up assessments at various lengths post hospitalization. The researchers sampled 174 mothers whose children (ages 2-7 years old) were unexpectedly hospitalized in the pediatric intensive care units (PICUs) of 2 children's hospitals.

c. Outcome(s): Research Results: These mothers received educational and social support using a tri-phased educational and behavioral interventional program when their critically ill children were admitted to these PICUs. The findings of this study indicated that of the mothers that were participants in such projects experienced improved maternal functionality and emotional-coping outcomes, which in turn improved the health outcomes of children.

d. Significance to Nursing and Patient Care: The study's data supports that nursing can aid in assessing maternal functionality during times of their children's acute illness to improve maternal and child outcomes.

Osborne $\mathrm{C}$ et al. ${ }^{23}$ Family structure transitions and changes in maternal resources and well-being.

1. Summary of Article: This article examined data from a family and child well-being study in order to examine if instability factors of family are associated with changes in perceived psychosocial support, maternal depression symptoms, and parenting stress amongst mothers with young children.

2. Research Elements: Design, Methods, Population, Strengths, Limitations: This was a longitudinal birth cohort study of urban families, and employed several hierarchical linear models to gage the association of family structure variables with mothers' access to psychosocial resources and psychological well-being. The data in this analysis is well suited because family complexity and instability are not uncommon, and unusually well measured.

3. Outcome(s): Research Results: The results of this study reveal that dissolution of relationships are associated with negative effects on maternal well-being, whereas a unity formation is related to positive or neutral effects on maternal well-being. The consequences of relationship dissemination may affect maternal functionality in both their family and individual roles. As a result, this has indirect influences on a child's well-being.

4. Significance to Nursing and Patient Care: This study can support continuous maternal depression screening through nursing assessments in pediatric clinics. The nursing profession must understand that maternal depression can occur frequently outside of the postpartum window well into a child's life. Psychosocial variables change and this can impact maternal health throughout the lifespan.
According to Schor $\mathrm{E}^{24}$ pediatrics: report of the task force on the family.

1. Summary of Article: This article supports that families are the most central unit of enduring influence in a child's life. Parents are also of great importance in pediatric care. The health of children can be indirectly linked to their parents' holistic, physical and psychosocial health and also to their parenting practices. The data shows there is a higher incidence of behavior problems among children and this attests to the inability of some families to cope with the toxic stressors that they are experiencing and their need for assistance. When a family's distress finds its voice in a child's symptoms, pediatricians are often parents' first source for help.

2. Research Elements: Design, Methods, Population, Strengths, Limitations: A task force found that an abundance of research is established about family functioning, psychosocial circumstances and the effects on children. With this knowledge, the provision of pediatric care is needed that promotes empowered families and good outcomes for children. The task force refers to this type of care as "family-oriented care" and strongly endorses policies and procedures that promote the adoption of this multi-generational approach to pediatric care.

3. Outcome(s): Research Results: The task force, that was to address pediatrics, decided to frame its deliberations and recommendations around family functionality and how various family factors construct and influence both parenting and child health.

4. Significance to Nursing and Patient Care: This is important to nursing because it helps to construct our assessments and interventions in a more patient centered way.

Wells et al. ${ }^{25}$ Community-partnered cluster-randomized comparative effectiveness trial of community engagement and planning or resources for services to address depression disparities.

a. Summary of Article: Quality improvement projects related to depression in primary care clinics can improve outcomes relative to primary preventative care. Another notion of this data includes that community-based health and social related partnerships also support clients in under-served communities. The data compares the effectiveness of Community Engagement and Planning and Resources for Services to facilitate the implementation of quality initiatives related to depression and the effects on clients' mental health-related quality of life and resource use.

b. Research Elements: Design, Methods, Population, Strengths, Limitations: Matched programs from health, social and other community service sectors were randomized to community engagement and promoting inter-agency collaboration of resources for services to implement depression QI toolkits in large inner-cities in California.

c. Outcome(s): Research Results:CEP was more effective than RS at improving mental health, quality of life, increasing physical activity, reducing risk factors related to homelessness, reduced the number of hospital admissions related to mental and behavioral health and medication visits among specialists. These improvements were made while increasing depression visits with primary care providers for depression and enhanced the number of people who used faith-based programs. The data reported that the use of antidepressants, employment, and total contacts were not significant. 
d. Significance to Nursing and Patient Care: Data from these services can help the nursing profession become more of a vital participant of Community Engagement and Planning. Nursing care extends well outside the walls of acute care hospitals.

Whittemore et al. ${ }^{26}$ Psychological experience of parents of children with type 1 diabetes: a systematic mixed-studies review.

a. Summary of Article: The purpose of this research review was to describe the occurrence of mental health distress in parents with children that have a chronic illness such as type 1 diabetes. The study examined the relationship between parental psychological distress and health outcomes. The researchers also took a close look at the experiences the parents' faced psychologically while having a child with chronic illness and determined the implication research and clinical support.

b. Research Elements: Design, Methods, Population, Strengths, Limitations: This study used a review of systematic mixedstudies. The study was undertaken to review the quantitative and qualitative research on the experiences of parents whose child has a chronic illness, in this case, type 1 diabetes mellitus. A total of 34 articles met criteria for inclusion and were used to help evaluate research.

c. Outcome(s): Research Results: This study reflects that the psychological distress of parents that have children with a chronic illness, regardless of definition, was associated with increased numbers of self-reported stress and depressive symptoms by children, more problematic child behavior, and lower levels of quality of life. Parental psychological distress also had counterproductive effects on diabetes management. Themes of the qualitative synthesis indicated that parents perceived that diabetes was a diagnosis that is difficult to manage and that contributes to family disruption. The suggested that adjustment to disease management occurred over time; however, and but ongoing stress was reported.

d. Significance to Nursing and Patient Care: These findings can direct nurses to stay attune to the maternal well -being when assessing their children with chronic diseases like T1DM at well checks.

\section{Acknowledgments}

None.

\section{Conflicts of interest}

The authors declare there is no conflict of interests.

\section{Funding}

None.

\section{References}

1. Swick Slover K. Nursing capstone projects bring students knowledge, skills together to help communities. 2006.

2. Gagliardi A, Honigfel L. Addressing maternal depression: Opportunities in the pediatric setting. IMPACT; 2008.

3. Bernard-Bonnin, A. C. (n.d.) Maternal depression and child development.

4. http://nursingplanet.com/health_promotion_model.html

5. Atkins R. Self-efficacy and the promotion of health for depressed single mothers. Men Health Fam Med. 2010;7(3):155-168.
6. Sohr-Preston SL, Scaramella LV. Implications of timing of maternal depressive symptoms for early cognitive and language development. Clin Child Fam Psychol Rev. 2006;9(1):65-83.

7. Hall LA, Rayens MK, Peden AR. Maternal Factors Associated With Child Behavior. J Nurs Scholarsh. 2008;40(2):124-130.

8. Heneghan AM, Mercer M, DeLeone NL. Will mothers discuss parenting stress and depressive symptoms with their child's pediatrician? Pediatrics. 2004;113(3):460-467.

9. Yuan CT, Nembhard IM, Stern AF, et al. Blueprint for the dissemination of evidence-based practices in health care. Issue Brief (Commonw Fund). 2010;86:1-16.

10. Center on the Developing Child, Harvard University. Maternal depression can undermine the development of young children. Harvard University; 2009.

11. Knitzer J, Theberge S, Johnson K. Reducing maternal depression and its impact on young children toward a responsive early childhood policy framework. National Center for Children in Poverty 2. 2008.

12. Karen A E, Janet W Rich-Edwards, Karestan C Koenen. Maternal depression in the united states: nationally representative rates and risks. Journal of Women's Health. 2011;20(11):1609-1617.

13. Purposes of Evaluations (Plausibility, Probability, Adequacy) - (2013) Unite For Sight.

14. Sia JH, Leventhal JM, Northrup VS, et al. Markers of maternal depressive symptoms in an urban pediatric clinic. J Pediatr. 2013;162(1):189-194.

15. Bassani DG, Padoin CV, Phillip D, et al. Estimating the number of children exposed to parental psychiatric disorders through a national health survey. Adolescent Psychiatry Mental Health. 2009;3(6).

16. Barlow J, Coren E. Parent-training programs for improving maternal psychosocial health. Cochrane Database Syst Rev. 2011;1:CD002020.

17. Fortney JC, Enderle MA, Clothier JL, et al. Population level effectiveness of implementing collaborative care management for depression. Gen Hosp Psychiatry. 2013;35(5):455-460.

18. Hanks C. Health disparities research and service learning. Journal of Multicultural Nursing \& Health. 2003;9(3):18-23.

19. Heneghan AM, Silver EJ, Bauman LJ, et al. Do pediatricians recognize mothers with depressive symptoms? Do pediatricians recognize mothers with depressive symptoms? Pediatrics. 2000;106(6):1367-1373.

20. Houtrow AJ, Okumura MJ. Pediatric mental health problems and associated burdens on families. Vulnerable Child Youth Stud. 2011;6(3):222-233.

21. Jordal M, Wijewardena K, Olsson P. Unmarried women's ways of facing single motherhood in Sri Lanka - a qualitative interview study. $B M C$ Womens Health. 2013;13(5).

22. Melnyk BM, Alpert-Gillis L, Feinstein NF, et al. Creating opportunities for parent empowerment: program effects on the mental health/coping outcomes of critically ill young children and their mothers. Pediatrics. 2004;113(6):e597-e607.

23. Osborne C, Berger LM, Magnuson K. Family structure transitions and changes in maternal resources and well-being. Demography. 2013;49(1):23-47.

24. Schor EL. Family pediatrics: report of the task force on the family. Pediatrics. 2003;111(6 Pt 2):1541-1571.

25. Wells KB, Jones L, Chung B, et al. Community-partnered clusterrandomized comparative effectiveness trial of community engagement and planning or resources for services to address depression disparities. J Gen Intern Med. 2013;28(10):1268-1278.

26. Whittemore R, Jaser S, Chao A, et al. Psychological experience of parents of children with type 1 diabetes: a systematic mixed-studies review. Diabetes Educ. 2012;38(4):562-579. 